\title{
Minimum Quality Standards and consumers' information
}

Paolo G. Garella and Emmanuel Petrakis Department of Economics, University of Bologna and

Department of Economics, University of Crete

December 15, 2004 


\begin{abstract}
The literature so far has analyzed the effects of Minimum Quality Standards in oligopoly, using models of pure vertical differentiation, with only two firms, and perfect information. We analyze products that are differentiated horizontally and vertically, with imperfect consumers information, and more than two firms. We show that a MQS changes the consumers' perception of produced qualities. This increases the firms' returns from quality enhancing investments, notwithstanding contrary strategic effects. As a consequence, MQS policies may be desirable as both, firms and consumers, can gain. This contrasts with previous results in the literature and provides a justification for the use of MQS to improve social welfare.
\end{abstract}

JEL: L0, L5

Keywords: Regulation, Minimum Quality Standards, Oligopoly, Product Differentiation, Asymmetric Information 


\section{Introduction ${ }^{1}$}

The quality of goods is at the center of much policy debate. In Europe, quality certification and quality standards have become a matter of increasing concern for firms over the years, along with the completion of the common market and the implementation of uniform trading rules. In many industries, ranging from passenger transport, to food, or children toys, quality and safety regulation are also intertwined and almost indistinguishable. Also, it is often claimed that consumers have to choose what to buy under less than full knowledge of the quality of goods; and in many cases qualities are difficult to ascertain even by experts equipped with the necessary tools. The present paper deals with mandatory Minimum Quality Standards (MQS) and studies their effects on product qualities and on welfare in an oligopolistic industry where buyers have less than full information about the quality of goods. Unlike the present paper, the existing theory of MQS is largely and almost uniquely based upon models of oligopolistic competition with pure vertical product differentiation. These models, derived from the seminal paper by Gabszewicz and Thisse (1979), share some well-known typical features. First, the firms' products differ only in one respect, the vertical quality dimension, which is observable and which, if increased, leads to higher utility for each consumer. Second, in a duopoly, the rival firms try to differentiate their products, in order to relax price competition (Shaked and Sutton (1981). This implies that one of the two producers assigns to its product a low level of quality, even if it costs nothing to increase it. Third, once a MQS is introduced at a level that lies between the low and the high unregulated quality levels (Ronnen 1991), the equilibrium price of goods and the equilibrium profits of firms decrease. Indeed, since the standard reduces the distance between the quality levels of the two firms, more intense price competition brings forth lower prices. This, by the way, also explains why aggregate profits fall. In this vein of the literature, other papers (Crampes and Hollander 1995, Scarpa 1998, Valletti 2000, Jinji and Toshimitsu 2002), explore the issues of consumers' and firms' gains and losses, and of quality changes, always based on models of pure vertical differentiation.

Another noteworthy common trait of the literature on MQS is that perfect information is generally assumed. This is at odds with the very beginning of explanations of MQS-like practices (Leland (1979), where, absent strategic interplays between producers, these are interpreted as corrections

\footnotetext{
${ }^{1}$ We wish to thank participants at the ASSET meeting 2004 in Barcelona for helpful comments. The first author thanks the University of Crete for its hospitality during some phases of this research project, started under Marie Curie fellowship MCFI-2002-00153.
} 
to a "lemon" phenomenon. In a way, a MQS in Leland (1979) has the effect of cutting away the lowest tail of a distribution of qualities, thereby increasing the average marketed quality in a competitive setting. Roughly speaking, the present paper is in the spirit of this interpretation of MQS, although the effects on consumers' expected quality here has a feedback on the firms' behavior in a strategic setting.

As to the results obtained in the literature on MQS in duopoly and under perfect information, some remarks are in order. The predicted decrease in firms' profits may be consistent with some real world cases, but it appears to contrast with other cases in which firms, or association of producers, voluntarily agree to impose Minimum Quality Standards or, in a similar way, quality certification standards. Documented instances can be found, e.g., in agriculture (for instance, Ferguson and Carman (1999) report how the California kiwifruit producers agreement to a standard allowed them to withstand competition from New Zealand). Furthermore, the assumption that consumers are perfectly informed prevents the applicability of results on consumer welfare to situations where MQS should be introduced because of concerns about imperfect consumers' information.

As to the differentiation feature, it has already been noted (Garella $2003),{ }^{2}$ that in a model with perfect information the effects of MQS on quality levels, are ambiguous if goods are differentiated also horizontally, as in the Hotelling (1929) linear city. The average quality in the market is found to be possibly decreasing because quality competition between firms is characterized by downward sloping reaction functions, so that in a duopoly a forced increase in quality due to the MQS by the low quality firm leads to a lowered quality by the high quality firm. This feature of reaction function is preserved in the model presented below, although the horizontal quality differentiation is represented here according to the so-called "Dixit-Spence" (or "Bowley") model and not according to a spatial model (Spence 1976, Dixit 1979, Bowley 1924).

The model we use is also characterized by imperfect information. Each consumer is assumed to receive some evidence about the utility that he shall derive by consuming the good. This piece of information can be alternatively interpreted as relating to the goods' true quality level, which may differ by what is written on the product "label", or as relating to the carefulness with which the good is manufactured, which may lead to failures of the good or of some of its components. This piece of information is in

\footnotetext{
${ }^{2}$ Perverse quality reactions to the standard had also been found by Scarpa (1995) under pure vertical differentiation.
} 
effect amenable to an idiosyncratic disturbance on the quality observation of the single consumer. The consumer's willingness to buy is affected by this piece of information received, and we exclude from the analysis any sort of signaling or certification activity by firms that may cancel the effect of this information.

Firms choose quality levels at the first stage of a game and at the second and final stage they choose prices. Qualities are costly, so that higher quality implies higher production costs.

The results that we obtain may help explain why MQS policies are used. In a model where firms are symmetric, the equilibrium qualities always improve. This is an important result. Indeed, in spite of the firms' reaction functions in quality being downward sloping, so that strategic effects on quality are negative, the effect of a MQS is that of reducing consumers' uncertainty about the quality of the two goods. This positive countervail effect leads to an increased willingness to pay for quality by consumers and to an increased effort in producing good quality by the firms. We also find that firms' profits increase, a result that would explain the use of quality certification by (far-sighted) producers' associations and the producers' willingness to accept MQS policies.

The effects on consumers' welfare are the most difficult to assess. Consumers that receive a correct information about goods coexist in the model with consumers that receive information that the goods are of lower quality than it is true. This second type of consumer is more willing to pay for the goods when MQS are introduced, because the range of quality uncertainty is reduced. However, this implies that firms are confronted with higher average demand and therefore will increase their equilibrium prices. This price effect hurts the perfectly informed consumers. Consumer's expected surplus can be increased, unless the signals that consumers receive about qualities are good with very large probability. Furthermore, too strong strategic effects in the quality game (namely high substitutability between the goods) also reduce the positive effects on consumers' welfare.

Our model has the advantage of allowing an analysis for a number of firms larger than two. The signs of the impact on qualities, profits, and welfare of MQS are confirmed for the case with $n$ firms. The unregulated quality level decreases with the number of firms, but the quality improvement obtained by a MQS increases, as the negative weight of the strategic effects seem to fade away with an increase in the number of competitors. 


\section{The Model}

In a market for differentiated goods, two firms compete by choosing the quality level of their products and their prices. These choices are modelled as a two stage game where at each stage moves are simultaneous.

The population of consumers is composed by individuals with identical tastes, summarized by the (gross) utility function

$$
U\left(x_{1}, x_{2}\right)=\left(\alpha+s_{1}\right) x_{1}+\left(\alpha+s_{2}\right) x_{2}-\left(x_{1}^{2}+x_{2}^{2}+2 \gamma x_{1} x_{2}\right) / 2+m
$$

where $x_{i}$ represents the quantity of good $i$ bought by the representative consumer, for $i=1,2$, and $m$ is the respective quantity of the "composite good". Further, $s_{i}$ represents a quality parameter for good $i$, while the parameter $\gamma$ is related to the degree of substitutability between the two goods and is restricted to lie in the interval $[0,1]$. When $\gamma=0$ the goods are independent and when $\gamma=1$ they are perfect substitutes. Further, $p_{1}$ and $p_{2}$ denote the unit prices for the respective goods and the price of the composite good is normalized as to be equal to 1 . The vector $p$ is $p=\left(p_{1}, p_{2}\right)$.

Under perfect information, maximization of utility with respect to $x_{1}$ and $x_{2}$ gives the (inverse) demand functions for the representative consumer, $p_{i}=\left(a+s_{i}\right)-x_{i}-\gamma x_{j}$, for $i, j=1,2$, and inverting we obtain the demand functions,

$$
x_{i}\left(p, s_{i}, s_{j}\right)=\frac{\alpha(1-\gamma)+\left(s_{i}-\gamma s_{j}\right)-p_{i}+\gamma p_{j}}{1-\gamma^{2}}, \text { for } j \neq i .
$$

For the sake of simplicity we normalize henceforth the mass of consumers to $N=1$.

Under imperfect information, each consumer receives a private signal about the quality of the good (as after imperfect inspection). The consumer's signal about good $i$ is denoted $\sigma_{i}$. Consumers in proportion $\mu$ receive a correct signal about good $i, \sigma_{i}=s_{i}$. While consumers in proportion $1-\mu$ receive the wrong signal $\sigma_{i}=s_{0}<s_{i}$. Messages are independent draws. Therefore the expected proportion of consumers that receive the correct information about the two qualities is $\mu^{2}$, while $(1-\mu)^{2}$ are expected to receive a wrong information about both goods, and $2 \mu(1-\mu)$ are expected to receive a wrong information about at least one of the two goods. This set up is a simplified, and therefore more manageable, account of what could be a more general environment ${ }^{3}$.

\footnotetext{
${ }^{3}$ For instance, true quality of each good results from (i) producer's investment in quality,
} 
Demand for good 1 by $N=1$ ex-ante identical consumers is:

$$
\begin{aligned}
q_{1}= & \mu^{2} x_{1}\left(p, s_{1}, s_{2}\right)+\mu(1-\mu) x_{1}\left(p, s_{1}, s_{0}\right)+ \\
& (1-\mu) \mu x_{1}\left(p, s_{0}, s_{2}\right)+(1-\mu)^{2} x_{1}\left(p, s_{0}, s_{0}\right) .
\end{aligned}
$$

A similar expression holds for good 2. From the notation it should be clear that, for instance, $x_{1}\left(p_{1}, p_{2}, s_{1}, s_{0}\right)=\frac{\alpha(1-\gamma)+\left(s_{1}-\gamma s_{0}\right)-p_{1}+\gamma p_{2}}{1-\gamma^{2}}$. Therefore, after some manipulation, the (expected) demand function under imperfect information, $q_{i}$, is given by:

$$
q_{i}\left(p_{i}, p_{j}, s_{i}, s_{j}, s_{0}, \mu\right)=\frac{(1-\gamma)\left[\alpha+s_{0}(1-\mu)\right]+\mu\left(s_{i}-\gamma s_{j}\right)-p_{i}+\gamma p_{j}}{1-\gamma^{2}}
$$

In order to simplify notation, let $w=(1-\gamma)\left[\alpha+s_{0}(1-\mu)\right]$, while $z_{i}=\mu\left(s_{i}-\gamma s_{j}\right)+w$, for $i \neq j$. Then, substituting into (3), we obtain:

$$
q_{i}=\frac{z_{i}-p_{i}+\gamma p_{j}}{1-\gamma^{2}}, \text { for } i, j=1,2 \text { and } j \neq i .
$$

This is a linear demand function, that depends upon the qualities, $s_{1}$ and $s_{2}$, upon the information precision, $\mu$ and $s_{0}$, and upon prices.

We assume that each one of the two firms produces only one of the two products; further, that its cost of producing quantity $q_{i}$ of good $i$ is given by:

$$
C_{i}\left(q_{i}, s_{i}\right)=c q_{i}+s_{i}^{2}
$$

As it can be seen we maintain the hypothesis that variable costs are independent of quality, while fixed costs are increasing, at an increasing rate, in quality. To simplify the exposition we assume the quality-independent marginal cost to be $c=0$. Then in the last stage, i.e. in the price competition stage, firm $i$ solves the maximization program

$$
\max _{p_{i}} \pi_{i}=p_{i}\left(\frac{z_{i}-p_{i}+\gamma p_{j}}{1-\gamma^{2}}\right)-s_{i}^{2}
$$

determining its average quality, $s$, and (ii) a disturbance, $\varepsilon$, generated by a random process. Firms could observe but not credibly reveal $\varepsilon$. Consumers would know its distribution. Through "inspection" some consumers would get to perfectly know $\varepsilon$. Some other would only learn that $\varepsilon$ is in some range (e.g. below a threshold level). 
where $s_{1}$ and $s_{2}$ are given as first stage choices. The best reply function for firm $i$ as a function of the rival's price $p_{j}$ is $p_{i}\left(p_{j}\right)=\frac{z_{i}}{2}+\frac{\gamma}{2} p_{j}$. Therefore, by solving the system of first order conditions, we obtain the Nash equilibrium prices, in terms of the original parameters,

$$
p_{i}^{*}=\frac{(2+\gamma)(1-\gamma)\left[\alpha+s_{0}(1-\mu)\right]+\mu\left[s_{i}\left(2-\gamma^{2}\right)-\gamma s_{j}\right]}{4-\gamma^{2}}
$$

And symmetrically for firm 2, by interchanging subscripts.

Obviously, $p_{1}^{*}=p_{2}^{*}$ holds only if $s_{1}=s_{2}$. Note that the equilibrium prices are increasing in $s_{0}$, the value of the lower signal for the wrongly informed consumers. The same is true for the equilibrium quantities that, from the first order conditions, are given by $q_{i}^{*}=p_{i}^{*} /\left(1-\gamma^{2}\right)$. Moreover, the program for firm $i$ at the first stage, is given by:

$$
\max _{s_{i}} \pi_{i}^{*}=\frac{1}{1-\gamma^{2}}\left[\frac{(2+\gamma)(1-\gamma)\left[\alpha+s_{0}(1-\mu)\right]+\mu\left[s_{i}\left(2-\gamma^{2}\right)-\gamma s_{j}\right]}{\left(4-\gamma^{2}\right)}\right]^{2}-s_{i}^{2} .
$$

This program admits a maximum for all $\gamma<0.9325 .{ }^{4}$ The best reply functions in qualities are downward sloping when the second order conditions are satisfied. This can be easily checked as, from the first order condition we get the best reply function for firm $i$ as

$$
s_{i}\left(s_{j}\right)=\mu\left(2-\gamma^{2}\right) \frac{(2+\gamma)(1-\gamma)\left[\alpha+s_{0}(1-\mu)\right]-\mu \gamma s_{j}}{\left(4-\gamma^{2}\right)^{2}\left(1-\gamma^{2}\right)-\mu^{2}\left(2-\gamma^{2}\right)^{2}},
$$

where the denominator is negative if the second order condition is satisfied. Therefore, qualities are strategic substitutes.

Exploiting symmetry, the Nash equilibrium qualities turn out to be:

$$
s_{i}=s^{*}=\frac{\mu\left(2-\gamma^{2}\right)\left[\alpha+s_{0}(1-\mu)\right]}{(2-\gamma)^{2}(1+\gamma)(2+\gamma)-\mu^{2}\left(2-\gamma^{2}\right)}, i=1,2
$$

Note that for $s_{0}<s^{*}$ to hold, the following condition should be satisfied:

$$
\frac{s_{0}}{a}<\frac{\mu\left(2-\gamma^{2}\right)}{(2-\gamma)^{2}(1+\gamma)(2+\gamma)-\mu\left(2-\gamma^{2}\right)},
$$

\footnotetext{
${ }^{4}$ While for each $\gamma \geq 0.9325, \mu$ should be smaller than $\mu_{c}(\gamma) \equiv\left(4-\gamma^{2}\right) \sqrt{\left(1-\gamma^{2}\right)} /(2-$ $\left.\gamma^{2}\right)$, with $d \mu_{c} / d \gamma<0$ and $\mu_{c}(1)=0$.
} 
i.e. the quality perceived by the uninformed consumers should be sufficiently low. In the sequel, we shall assume that (11) holds.

We can then state the following results.

Lemma 1. The equilibrium quality level in an unregulated duopoly is decreasing in $\gamma$ and is therefore highest when the goods are independent.

Proposition 1. The introduction of a $M Q S$, denoted $Q$, such that $s_{0}<Q$, leads to an increase in the equilibrium quality level.

Note that the increase in quality is obtained also for values of the standard that lie below the unregulated quality level $s^{*}$. Indeed, this is the situation that one may have in mind. What about the effect of $\gamma$ on the effectiveness of a MQS policy? From (10), we get,

Lemma 2. The MQS effectiveness on quality is decreasing in the degree of substitutability between the two products.

Indeed, one has that $\frac{d^{2} s}{d s_{0} d \gamma}<0^{5}$. The policy instrument is most effective for independent goods, where there are no strategic effects. This confirms that strategic effects decrease the marginal rentability of investments in quality while direct effects tend to increase it.

\section{$3 \quad$ Welfare Analysis}

The welfare analysis of the effects of MQS depend upon the effects on quality, price, quantity. We can start from the following result:

Proposition 2. Equilibrium price, quantities, and profits are increased if a standard, $Q$ such that $s_{0}<Q<s^{*}$, is introduced.

Note however that from (7) and (10) one obtains that the hedonic price, namely the price-quality ratio, is given by,

$$
\frac{p^{*}}{s^{*}}=\frac{\left(4-\gamma^{2}\right)\left(1-\gamma^{2}\right)}{\left(2-\gamma^{2}\right) \mu}
$$

Thus, the hedonic price does not depend on $s_{0}$ and is inversely related to $\mu$ and $\gamma$.

The firms' equilibrium profits always increase due to the introduction of the standard. Consumers that are receiving $\sigma=s_{0}$, bad information, about one or both products revise their expectations if a standard prevails. Their

\footnotetext{
${ }^{5}$ The cross second derivative is $\frac{d^{2} s}{d s_{0} d \gamma}=\frac{\mu(1-\mu)}{g(\cdot)^{2}}\left(2 \gamma^{5}-\gamma^{4}-8 \gamma^{3}+2 \gamma^{2}+8 \gamma-8\right)$, which is negative.
} 
willingness to pay is increased with respect to the no-standard case, and their demand for both goods shifts up.

The expected utility of consumers varies according to their expectation of qualities. We distinguish an ex-ante and an interim stage. At the first stage one can define the expected utility of a consumer that does not know which signals he shall receive. The second is the stage at which each consumers has received the individual signals $\sigma_{1}$ and $\sigma_{2}$. At the interim stage, the four groups of consumers obtain different (interim) expected utility. Obviously, a consumer does not know to which group he will be assigned and therefore $e x$ ante, or expected, utility is the expectation of the interim utilities, weighted by the appropriate probabilities, $(1-\mu)^{2}, \mu(1-\mu)$, and $\mu^{2}$.

It is easy to show that the interim utility of consumers that get the wrong information for both goods, and therefore would buy on the quality perception $s_{0}$ for both goods, is increased by the standard. The utility of those consumers that at the interim stage are perfectly informed is increased by the standard only in a region of the parameters, because of the price increase effect they pay for enjoying the equilibrium quality increase.

Consumers that receive a wrong signal about both goods, call it "group 1 " have utility function,

$$
U\left(x_{1}, x_{2}\right)=\left(\alpha+s_{0}\right)\left(x_{1}+x_{2}\right)-\left(x_{1}^{2}+x_{2}^{2}+2 \gamma x_{1} x_{2}\right) / 2+m-p_{1} x_{1}-p_{2} x_{2}
$$

Accordingly, the consumers with interim information that both products are of low quality gain from the introduction of a MQS.

Lemma 3. The interim expected utility of consumers receiving the wrong signal that both products are of bad quality is increased after the introduction of a MQS.

Let us now analyze the utility change of consumers that receive the signal that both goods are of high quality. Group 4: consumers that are perfectly informed.

$$
U(h)=\left(\alpha+s^{*}-p_{1}\right) x_{1}+\left(\alpha+s^{*}-p_{2}\right) x_{2}-\left(x_{1}^{2}+x_{2}^{2}+2 \gamma x_{1} x_{2}\right) / 2+m
$$

Lemma 4. The interim expected utility of consumers receiving the true signal about products is decreased after the introduction of a MQS, except if the two inequalities, $\gamma>\sqrt{(2-\sqrt{2})}$ and $\mu>\left(4-\gamma^{2}\right) \frac{1-\gamma^{2}}{2-\gamma^{2}}$, hold together. 


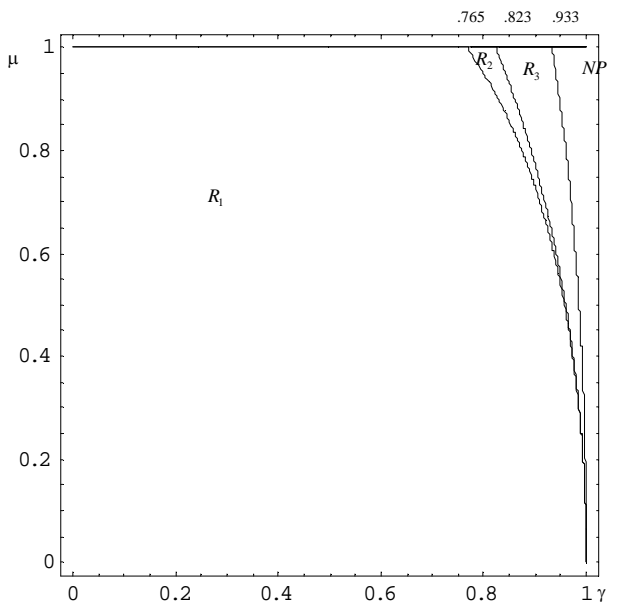

Fig. 1: In $R_{1}$ and $R_{2}$ TW increases always with MQS; in $R_{1}$ CS increases always with MQS; $\mathrm{NP}=$ non-permissible region

Figure 1:

Finally, we have no clear-cut result about those consumers (group 2 and group 3) who get a signal that only one of the qualities is $s_{0}$. Notice only that the relative magnitude of this group decreases monotonically as the product $\mu(1-\mu)$ is increased and tends to 0 for $\mu$ tending to 1 (or 0 ).

However, by evaluating the weighted sum of the interim surpluses we obtain the expected consumer surplus for a consumer. We then get the following result for total and for consumer welfare.

Proposition 3. (a) Total expected welfare in the industry is increased by the introduction of a standard over a compact set in the $(\gamma, \mu)$ space with measure larger than 92\% of the region of admissible values of $(\gamma, \mu)$ pairs; in particular, such that either $\gamma<0.823$ or $\gamma>0.823$ and $\mu<\mu^{w}(\gamma)$ where $\mu^{w}(\gamma)$ is a decreasing function of $\gamma$ which takes value 1 at $\gamma=0.823$. (b) Expected consumer surplus is increased by the introduction of a standard for a region in the $(\gamma, \mu)$ space larger than $90 \%$ of all values of $(\gamma, \mu)$ pairs; in particular, such that either $\gamma<0.765$ or $\gamma>0.765$ and $\mu<\mu^{c}(\gamma)$ where $\mu^{c}(\gamma)$ is a decreasing function of $\gamma$ which takes value 1 at $\gamma=0.765$. 
The proposition is illustrated in Figure 1, where the region denoted $R_{1}$ represents the region where total consumer surplus is increasing, while the region formed by the sum of $R_{1}$ and $R_{2}$ is the region over which total welfare in the industry is increasing. As for ex-post utility one has two possibilities to consider. The first is that a consumer never gets to know the true quality and remains with the information given at the interim stage. Then the sum over all consumers of the ex-post utility would be equal to the expected utility, if the population of consumers is large enough. In that case the results in proposition 3 would apply.

The second possibility for ex-post utility is that a consumer ex-post gets to know the quality of the good he consumes. This implies that a consumer belonging, say, to the group that receives the wrong information about both products buys a quantity of each good given by $x_{\ell}\left(p^{*}, Q, Q\right)=\frac{(\alpha+Q-p)}{1+\gamma}$, where $Q$ is the level of the standard. Ex post they get to know the true quality and re-evaluate their utility according to the quantities they have bought. We have no clear-cut results over the behavior of consumer surplus in the parameter space, but we can assert that total welfare in the industry follows a similar pattern to that of expected total surplus.

\section{Extension to more than 2 firms}

Let $x=x_{1}, \cdots, x_{n}, s=s_{1}, \cdots, s_{n}$ and $p=p_{1}, \cdots, p_{n}$ be the vectors of the quantities and prices of the $n$ differentiated goods produced in the industry. The utility function of the representative consumer, setting $m=0$ to shorten it, is given by

$U(x, s)=\sum_{i=1}^{n}\left(a+s_{i}\right) x_{i}-\frac{\left(x_{1}^{2}+\cdots x_{n}^{2}+2 \gamma\left(x_{1} x_{2}+\cdots+x_{n-1} x_{n}\right)\right)}{2}-\sum_{i=1}^{n} p_{i} x_{i}$

namely

$$
U(x, s)=\sum_{i=1}^{n}\left(a+s_{i}\right) x_{i}-\frac{\left(\sum_{i=1}^{n} x_{i}^{2}+2 \gamma \sum_{i=1}^{n} \sum_{j=1, j \neq i}^{n} x_{i} x_{j}\right)}{2}-\sum_{i=1}^{n} p_{i} x_{i}
$$

As a result, under perfect information the inverse demand function for firm $i$ is

$$
p_{i}=\left(a+s_{i}\right)-x_{i}-\gamma x_{-i}, \text { where } x_{-i}=\sum_{j \neq i} x_{j}
$$


Then the demand function for firm $i$ is given by

$$
x_{i}=\frac{a(1-\gamma)+(1+\gamma(n-2))\left(s_{i}-p_{i}\right)+\gamma\left(p_{j}-s_{j}\right)}{(1-\gamma)(1+\gamma(n-1))},
$$

where $p_{-i}=\sum_{j \neq i} p_{j}$ and $s_{-i}=\sum_{j \neq i} s_{j}$.

Under uncertainty, i.e. when the probability of observing the true quality $s_{i}$ is $\mu$ (with $1-\mu$ the probability of believing that the quality is $s_{0}$ ), and the quality signals being independent draws, the demand function of firm $i$ (after manipulations) turns out to be surprisingly simple. Letting $[a+(1-$ $\left.\mu) s_{0}\right](1-\gamma)=V$ it is given by:

$$
q_{i}(p, s, n)=\frac{V+(1+\gamma(n-2))\left(\mu s_{i}-p_{i}\right)+\gamma\left(p_{-i}-\mu s_{-i}\right)}{(1-\gamma)(1+\gamma(n-1))} .
$$

Accordingly, the profit function can be written as $\pi_{n}=p q_{i}(p, s, n)$, and standard calculations show that the best price reply function is $p_{i}(n)=$ $\frac{V+\gamma p_{-i}+\mu s_{i}(1+\gamma n-2 \gamma)-\mu \gamma s_{-i}}{2(1+\gamma n-2 \gamma)}$ and the Nash equilibrium prices can be calculated as,

$$
p_{n}^{*}=\frac{V+\mu s_{i}(1+\gamma n-2 \gamma)-\mu \gamma s_{-i}}{2+\gamma n-4 \gamma} .
$$

One can show that the effect of a MQS on equilibrium quality is positive, by simply looking at the effects on the marginal benefit of an investment in quality, as follows. Let the equilibrium demand be $D_{n}^{*}$, then ${ }^{6}$ the equilibrium profits are

$$
\pi_{n}=p_{n}^{*} D_{n}^{*}-s_{i}^{2}
$$

This can be summarized as:

Proposition 4. In an oligopoly with $n>2$ firms, equilibrium quality and equilibrium profits are increased if a MQS higher than $s_{0}$ is introduced.

The analysis for consumers' welfare is more complex than for the case with two firms, but analogous results are obtained. As for profits, since quality is higher, price shall be higher at a symmetric solution. However, the quality investment will require additional resources and increase cost.

$$
{ }^{6} D_{n}^{*}=\frac{V-(1+\gamma(n-2)) p+\gamma n p+\mu\left((1+\gamma(n-2)) s_{i}-\gamma s_{2}\right)}{(1-\gamma)(1+\gamma(n-1))} .
$$


However, the effect on consumers' expectations about quality is the same as for the case with two firms: consumers' willingness to pay is increased for each true quality level. This is equivalent to an outward shift in demand function for each firm, and cannot be detrimental to profits.

The effect of raising $n$ on the equilibrium outcome is an interesting question. The following proposition states the main results

Proposition 5. Unregulated quality is decreasing in n, while the effectiveness of a MQS policy is increasing with $n$.

This conclusion again depends upon the property that the intensity of the strategic effects fades away as the number of competitors increases.

\section{Conclusions}

We have reconsidered the effects of Minimum Quality Standards policies in oligopoly, introducing imperfect information, and brand differentiation. The analysis above has shown that both, firms and consumers, may gain from the introduction of mandatory quality standards. This is a novel result. The key to the result is in the updating by consumers of their expectations about the true quality of the good when a MQS is introduced. This updating may be obtained, more generally, in models where the information acquisition process is less specific. For instance, one may assume that consumers observe a stochastic signal that is centered around the true quality. The MQS would be effective then as far as it cuts from below the support of the distribution of the signal.

This shows that the policy implications and the rationale for the observed use of MQS and of mandatory certification policies markedly differ in the two cases of perfect and imperfect information, because in the first case the firms reaction to the standard does not depend upon the change in consumers' expectations. Since in the real world quality concerns are strong also where consumers have imperfect information and where they have difficulties in assessing the way in which goods are manufactured, our analysis seems justified. 


\section{REFERENCES}

Bowley A.L., 1924, The Mathematical Groundwork of Economics, Oxford University Press, Oxford.

Crampes, C. and Hollander A., 1995, Duopoly and quality standards, European Economic Review 39, 71-82.

Dixit A., 1979. A model of duopoly suggesting a theory of entry barriers, Bell Journal of Economics, 10, 20-32.

Ecchia G. and L. Lambertini, 1997. Minimum Quality Standards and Collusion, Journal of Industrial Economics 44, 101-

Ferguson, C. and H. Carman, 1999, Kiwifruit and the "Lemon" problem: do Minimum Quality Standards work?, Agribusiness Forum Papers, www.ifanna.org/ conferences/9/1999_forum_papers.htm

Gabszewicz-Jaskold J. and J.-F. Thisse, 1979. Price competition, qualities and income disparities, Journal of Economic Theory 20, 340-359.

Garella P.G. 2003, The Effects of minimum quality standards: better or worse products? WP 484 Department of Economics (www.dse.unibo.it/ ricerca) September 2003

Hotelling, H. 1929, Stability in competition, Economic Journal 39, 41-57.

Jinji N. and T. Toshimitsu 2002, Minimum Quality Standards under asymmetric duopoly with endogenous quality ordering: A note, Journal of Regulatory Economics, 26, 189-199.

Leland, H.E. 1979. Quacks, Lemons, and Licensing: A theory of Minimum Quality Standards, Journal of Political Economy 87, 1328-1346.

Lutz, S.; Lyon T.and J.W.Maxwell 2000. Quality leadership when regulatory standards are forthcoming, Journal of Industrial Economics, 48, $331-48$.

Maxwell, J.W. 1998. Minimum quality standards as a barrier to innovation, Economics Letters, 58, 355-60.

Ronnen, U. 1991. Minimum quality standards, fixed costs, and competition, Rand Journal of Economics 22, 490-504. 
Scarpa, C. 1998. Minimum quality standards with more than two firms, International Journal of Industrial Organization 16, 665-676.

Shaked A. and J. Sutton 1982, Relaxing price competition through product differentiation, Review of Economic Studies 49, 3-14.

Spence A.M. 1976, Product Differentiation and Welfare, American Economic Review, 66, 407-414.

Valletti, T. M. , 2000, Minimum Quality Standards under Cournot competition, Journal of Regulatory Economics, 18, 235-245. 


\section{Appendix}

\section{Proof of Lemma 1}

Letting $\beta_{0}=\alpha+s_{0}(1-\mu)$ and taking the derivative of (10), we get $d s^{*} / d \gamma=$ $\frac{\mu \beta_{0}}{g(\gamma, \mu)}\left(2 \gamma^{5}-\gamma^{4}-8 \gamma^{3}+2 \gamma^{2}+8 \gamma-8\right)$ where $g(\gamma, \mu)=(2-\gamma)^{2}(1+\gamma)(2+$ $\gamma)-\mu^{2}\left(2-\gamma^{2}\right)$. Therefore, the effect of $\gamma$ on quality is signed as negative if the term at the numerator is positive, which is true for all values of $\gamma$ in the $[0,1]$ interval.

\section{Proof of Proposition 1}

If $Q>s_{0}$, consumers who receive the wrong quality signal will update it to $s_{0}=Q$. Notice from (10) that $\left(d s^{*} / d s_{0}\right)>0$, so that the effect of an increase in the standard on equilibrium qualities is positive.

\section{Proof of Proposition 2}

Substituting (10) into (7) and simplifying, we obtain

$$
p_{i}^{*}=p^{*}=\frac{\left(4-\gamma^{2}\right)\left(1-\gamma^{2}\right)\left[\alpha+s_{0}(1-\mu)\right]}{(2-\gamma)^{2}(1+\gamma)(2+\gamma)-\mu^{2}\left(2-\gamma^{2}\right)}, i=1,2
$$

Moreover, from the first order conditions, we know that $q_{i}^{*}=q^{*}=\frac{p^{*}}{1-\gamma^{2}}$. It is then clear that $\frac{d p^{*}}{d s_{0}}>0$ and $\frac{d q^{*}}{d s_{0}}>0$, so that both equilibrium prices and quantities are increasing in $s_{0}$ and therefore in the standard. Turning to profits, by the first order conditions, equilibrium profits are equal to $\frac{\left(p^{*}\right)^{2}}{1-\gamma^{2}}-\left(s^{*}\right)^{2}$. Substituting $p^{*}$ from (7) and $s^{*}$ from (10), we get after some manipulations:

$$
\pi^{*}=\frac{\left[\left(4-\gamma^{2}\right)^{2}\left(1-\gamma^{2}\right)-\mu^{2}\left(2-\gamma^{2}\right)^{2}\right]\left[\alpha+s_{0}(1-\mu)\right]^{2}}{\left[(2-\gamma)^{2}(1+\gamma)(2+\gamma)-\mu^{2}\left(2-\gamma^{2}\right)\right]^{2}}, i=1,2
$$

which is positive. Clearly, equilibrium profits are increasing in $s_{0}$, and thus increase due to the introduction of the standard.

\section{Proof of proposition 6 .}

Inverse quality, namely $\frac{1}{s^{*}}$ is equal to $\frac{\left((1-\gamma)(1+\gamma(n-1))(2+\gamma n-4 \gamma)^{2}\right)}{\left(\left(\left(a+(1-\mu) s_{0}\right)(1-\gamma)\right)\left(\mu(1+\gamma n-2 \gamma)^{2}\right)\right)}+\mu \frac{1-\gamma}{V}$, so that $\frac{d\left(1 / s^{*}\right)}{d n}$ is positive. Hence $\frac{d s^{*}}{d n}<0$. Quality as a function of $n$ shows the following first derivative: $\frac{d s^{*}}{d n}=\frac{x(n)}{V \mu(1+\gamma n-2 \gamma)^{3}}$, where $x(n)$ is a term that is independent of $s_{0}$. We know that $\frac{d s^{*}}{d n}$ is negative and therefore $\frac{d^{2} s^{*}}{d s_{0} d n}$ has the same sign (positive) as $\frac{d^{2} s^{*}}{d V d n}=-\frac{1}{V}\left(\frac{d s^{*}}{d n}\right)$. 